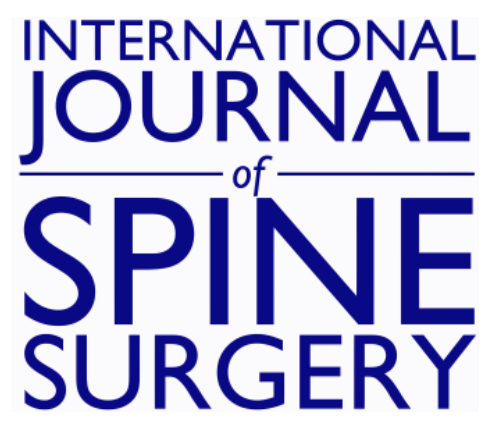

\title{
Percutaneous, Navigated Minimally Invasive Posterior Cervical Pedicle Screw Fixation
}

\author{
Domagoj Coric, Vincent J. Rossi, John Peloza, Paul K. Kim and Tim E. Adamson
}

Int J Spine Surg 2020, 14 (s3) S14-S21

doi: https://doi.org/10.14444/7122

http://ijssurgery.com/content/14/s3/S14

This information is current as of April 26, 2023.

Email Alerts Receive free email-alerts when new articles cite this article. Sign up at:

http://ijssurgery.com/alerts 


\title{
Percutaneous, Navigated Minimally Invasive Posterior Cervical Pedicle Screw Fixation
}

\author{
DOMAGOJ CORIC, MD,${ }^{1,2}$ VINCENT J. ROSSI, MD, ${ }^{1,2}$ JOHN PELOZA, MD,${ }^{3}$ PAUL K. KIM, MD, ${ }^{1,2}$ TIM E. \\ ADAMSON, MD ${ }^{1,2}$ \\ ${ }^{I}$ Carolina Neurosurgery and Spine Associates, Charlotte, North Carolina,${ }^{2}$ Atrium Health Musculoskeletal Institute, Charlotte, North Carolina, ${ }^{3}$ Center for Spine \\ Care, Dallas, Texas
}

\begin{abstract}
Background: Cervical pedicle screws provide significant biomechanical advantage but can be technically challenging and associated with morbid exposure. Improvements in intraoperative navigation guidance and instrumentation have made feasible this biomechanically robust, but technically challenging procedure. We present our initial experience with minimally invasive (MIS) percutaneous pedicle screw fixation in the cervical atlantoaxial and subaxial spine.

Methods: A retrospective review was performed on 27 cases that involved a novel MIS percutaneous cervical pedicle screw technique. Small lateral skin incisions were made bilaterally on the neck using intraoperative navigation guidance. Subsequently, navigated, percutaneous screws were placed using the Proficient Minimally Invasive System (PROMIS; Spine Wave, Shelton, CT). Computed tomography (CT)-guided navigation was used for cervical pedicle screw placement with subsequent placement of percutaneous rods.

Results: Indications for surgery included type II odontoid fractures, subaxial fracture dislocations and burst fracture, metastatic pathological burst fracture, and degenerative spondylosis with stenosis. There were 15 men and 12 women, with an average age 63.5 years. Follow-up ranged from 3 to 24 months (average $=16.7$ months). One screw was revised intraoperatively. Two patients $(7.7 \%)$ required reoperation, 1 patient required repositioning of a $\mathrm{C} 5$ pedicle screw, and 1 suffered a C7 body fracture. No nerve root injury, spinal cord injury, or vertebral artery injuries were reported.

Conclusions: Percutaneous cervical pedicle screw fixation is a feasible and safe technique when performed with CT-guided intraoperative navigation techniques. Cervical pedicle screw fixation provides a biomechanically superior construct in comparison with a lateral mass technique. In addition, the lack of paraspinal muscle disruption preserves important stabilizers of the posterior ligamentous complex and may reduce wound-healing issues in high-risk cases (eg, trauma patients). Although the current role for percutaneous instrumentation is relatively narrow, the advancement of MIS posterior cervical techniques may provide expanded opportunities in the future.
\end{abstract}

Special Issue

Keywords: cervical pedicle screws, minimally invasive, percutaneous, navigation

\section{INTRODUCTION}

Traditional open posterior approaches to the cervical spine are associated with significant approach-related morbidity secondary to denervation and atrophy of the paraspinal musculature and the disruption of the musculoligamentous complex. In addition, lateral mass instrumentation generally involves a relatively limited screw fixation, typically using $3.5 \times 12-\mathrm{mm}$ or $14-\mathrm{mm}$ screw sizes. ${ }^{1,2}$ Cervical pedicle screws were introduced to provide a biomechanically superior fixation. ${ }^{3-5}$ However, the significant muscle dissection associated with the lateral to medial trajectory exacerbates the significant approach-related morbidity associated with traditional open posterior cervical surgery. ${ }^{6-9}$ Moreover, the narrow pedicles of the subaxial cervical spine put adjacent vascular and neural structures at risk of breach. ${ }^{7}$

In 2006, the O-arm Imaging System was introduced (Medtronic, Minneapolis, MN); this was the first mobile, intraoperative 3-dimensional (3D) computed tomography (CT) imaging navigation system. This system provided enhanced workflow and registration advantages over its predecessors. The O-arm has resulted in rapid adoption of $3 \mathrm{D}$ CT-guided navigation in spinal surgery. ${ }^{10}$ Intraoperative $\mathrm{O}$-arm-based $3 \mathrm{D}$ navigation has allowed for increased accuracy of instrumentation placement as well as real-time virtual visualization of instru- 
ments. ${ }^{11}$ In addition, intraoperative imaging has allowed for immediate evaluation of hardware placement. This technology has resulted in new techniques due to the unique capabilities of these navigation systems. ${ }^{10}$

The navigated percutaneous cervical pedicle screw technique circumvents the drawbacks of open pedicle screws while achieving superior fixation when compared with standard lateral mass fixation. With the advent of advanced intraoperative CT navigation and minimally invasive (MIS) muscle-splitting techniques, the percutaneous cervical pedicle screw placement technique can be accomplished safely and effectively in select patients. $^{12,13}$ This MIS muscle-sparing technique allows for biomechanically superior lateral to medial trajectory with generally larger diameter and longer screws while minimizing exposure morbidity. We present our initial experience with purely percutaneous cervical pedicle screws and describe the radiographic outcomes and complication rate.

\section{METHODS}

A retrospective review was performed of our initial experience with 27 patients using a MIS percutaneous cervical pedicle screw technique. Patients were selected at the discretion of the treating surgeon, who took into account operative goals and anatomy favorable for use of this technique. These cases represented the initial experience and consecutive cases at 2 separate institutions. Three separate primary surgeons were involved. Included in this study were all patients who underwent percutaneous pedicle screw instrumentation. No patients who underwent percutaneous cervical pedicle screw fixation were excluded from the study. All patients had 2D and/or 3D intraoperative imaging with serial postoperative radiographs. All procedure-related intraoperative and postoperative adverse events were reported.

\section{Technique}

For the subaxial cervical spine, 2 lateral incisions were made paracentrally on the posterior neck over the spinal segments to be stabilized using intraoperative navigation (Stealth Station 8, Medtronic, Minneapolis, MN). Separate fascial incisions were used for each level to be instrumented.
For atlantoaxial fixation, bilateral 2-cm stab incisions were made approximately $5 \mathrm{~cm}$ from the midline, over the $\mathrm{C} 1$ lateral masses, and separate bilateral $2-\mathrm{cm}$ stab incisions were made approximately $4 \mathrm{~cm}$ from the midline at the $\mathrm{C} 6-\mathrm{C} 7$ level parallel to the extended angle of the $\mathrm{C} 2$ pars. A combination of sharp and blunt dissection was carried down through the fascia and muscle to the spine without the use of a retractor system. The Proficient Minimally Invasive System (PROMIS; Spine Wave, Shelton, CT) with reduction towers designed for use in the cervical spine was used for screw placement. The rods were then passed via the percutaneous technique. Postplacement intraoperative $\mathrm{CT}$ was performed to confirm accurate screw placement.

\section{Preoperative Evaluation}

All patients were evaluated with a preoperative cervical spine CT to measure the diameter of the pedicles under consideration of fixation. In patients with traumatic injury, a CT angiogram of the neck was also performed to evaluate for any preexisting vertebral artery injury or aberrant tortuosity that might affect operative planning. A cervical spine MRI was obtained as well to determine whether the pathology required anterior or posterior decompression in conjunction with percutaneous pedicle screw placement.

\section{Positioning}

The patient was placed in a Mayfield frame and positioned prone on Jackson table with a Mayfield attachment or cervical management system. The alignment of the cervicothoracic junction was maintained in a neutral position if fixating across the cervicothoracic junction. In addition, the head was maintained in a neutral position. When performing C1-C2 fixation, a slightly flexed position provided better access to the $\mathrm{C} 1$ lateral masses, but the patient was repositioned appropriately once screws were placed prior to rod placement. Taping the shoulders down allowed for access to the lateral neck. Sterile prep was performed in traditional fashion. It was of critical importance to prep extremely wide on the neck due to the unusually lateral-to-medial angle required for percutaneous cervical pedicle screw placement (Figure 1A). 

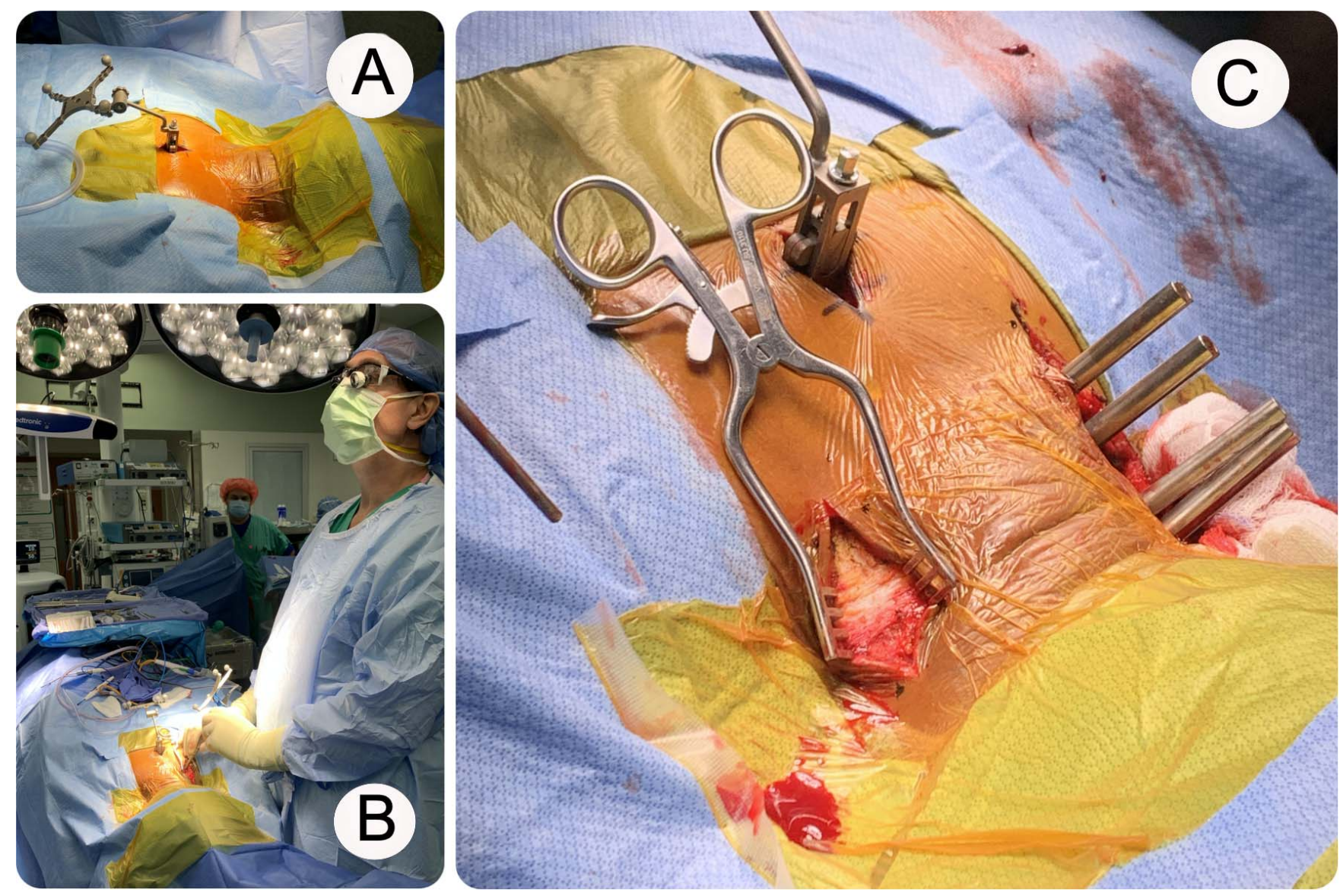

Figure 1. (A) Preparation, draping, and placement of spinous process clamp and navigation array for the subaxial percutaneous pedicle screw technique. (B) Drilling of a pilot hole along the trajectory of the pedicle. (C) Skin incision down to the layer of the investing fascia.

\section{Navigation}

Cone-beam $\mathrm{CT}$ intraoperative imaging was obtained using the O-Arm 2.0 (Medtronic, Minneapolis, $\mathrm{MN}$ ) for use with an intraoperative navigation system. For subaxial fixation, a fiducial was placed on a spinous process caudal to the lowest instrumented vertebra. This midline incision required for spinous process exposure was approximately $2.5 \mathrm{~cm}$ and involved very superficial release of the cervical fascia and paraspinal musculature to allow for clamp placement. The fiducial was unlikely to be obstructive even if unintentionally placed on the spinous process of a planned instrumented vertebrae, given the unusually wide angle of screw placement. The spinous process clamp provided more consistent accuracy, given small micromovements that may have occurred throughout the procedure, which can be worsened in cases of cervical instability. For atlantoaxial fixation, a fiducial attachment was placed directly on the Mayfield retractor.

\section{Screw Fixation}

The instrumentation system is designed to allow for true percutaneous pedicle screw placement in the cervical spine. Screws are available with various diameters $(3.8,4.2,4.5$, and $5.5 \mathrm{~mm})$ and with lengths ranging from 10 to $90 \mathrm{~mm}$. Screws were attached to a reduction tower system specifically designed for use in the cervical spine. Cobalt chrome or titanium rods with diameters of 3.5 or $4.0 \mathrm{~mm}$ were then placed via percutaneous technique.

\section{Technique: Subaxial Pedicle Screws}

Once the fiducial array had been placed on the spinous process of the lowest vertebral body to be instrumented and an intraoperative CT had been performed, the next step was planning the percutaneous incision. The navigated wand with an extended projection was used to establish the necessary entry point to provide the desired trajectory. Once each entry point was identified, a linear incision through the dermis was made down to the level of investing fascia along the course of 


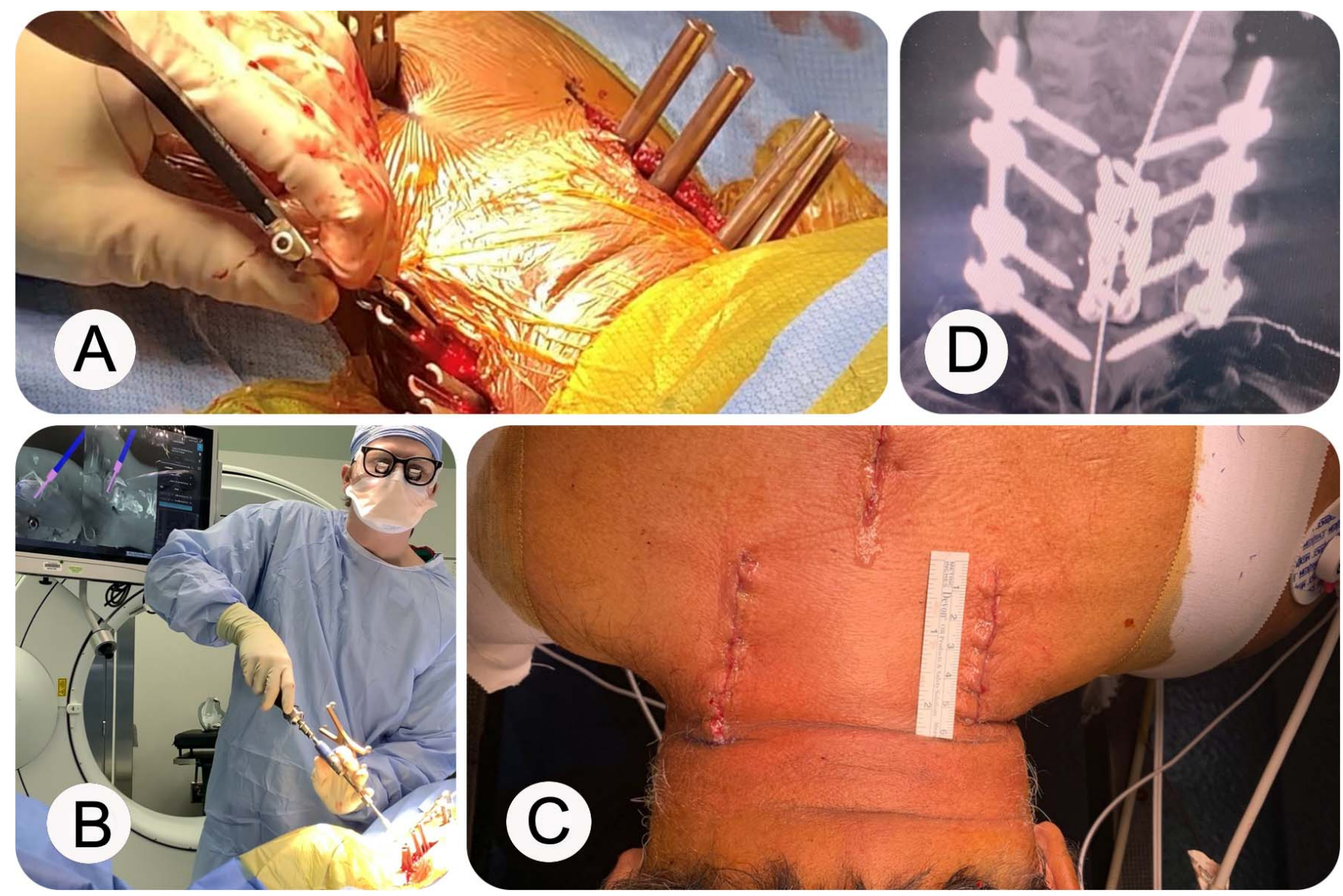

Figure 2. (A) Percutaneous placement of rod. (B) Pedicle screw placement. (C) Skin closure. (D) Final construct.

the entry points. This incision provided a more cosmetic closure as opposed to several small stab incisions, which could have been used as an alternative. A standard small, self-retaining Weitlaner retractor was used (Figure 1C). The navigated wand was again used to determine the percutaneous entry point through the fascia. Monopolar electrocautery was used to go down through the fascia, and the muscle fibers were split bluntly cranial caudally. Any deep fascia layers were released with the Bovie to allow for the placement of the navigated MH80 matchstick drill onto the lateral mass (alternatively, a navigated 2-mm burr could have been used) to drill a pilot hole. The navigated pilot hole allowed for efficient drilling, tapping, and screw placement with minimal skiving. The trajectory was confirmed and the navigated drill was advanced into the lateral mass along the axis of the pedicle (Figure 1B). If the pedicle was large enough in diameter, the drill was advanced at least midway through the pedicle. A 3.0-mm navigated tap was used for 3.5- and 4.0-mm screws, whereas a $4.0-\mathrm{mm}$ navigated tap was used for 4.5 - and $5.0-\mathrm{mm}$ screws. Navigation software was then used to select appropriate screw length and size. A navigated screw and reduction tower were then placed using navigation (Figure 2B).

After placement of pedicle screws on both sides, a second intraoperative CT was performed to confirm appropriate placement. A rod was then passed using the percutaneous technique and tested to confirm it was through all reduction towers (Figure 2A). Capture of the rod through each reduction tower was confirmed with a testing device that indicated the presence of the rod. The locking caps were placed and final tightened. Rod length was often confirmed visually or with direct palpation. If there was any concern or if additional alignment adjustments were desired, final intraoperative imaging was obtained with anterior-posterior and lateral fluoroscopy with the O-arm or C-arm (Figure 2D). The rod holder was then removed. Superficial cervical fascia were then closed with interrupted 2-0 resorbable sutures. Subcutaneous inverted 2-0 resorbable sutures were then placed for wound closure and topical skin adhesive was placed on the skin (Figure 2C). 


\section{Technique: C1-C2 fixation}

Either a C1-C2 transarticular screw or C1 lateral mass and $\mathrm{C} 2$ pars screw/rod fixation was achieved with this navigated, percutaneous posterior cervical technique. The fiducial array was placed on the Mayfield frame and intraoperative CT imaging was obtained. For a C1-C2 transarticular screw, the navigated wand was used to place a skin and fascial stab incision parallel to the extended angle of the $\mathrm{C} 2$ pars and in line with the cervical lateral masses. Bilateral stab incisions were made at approximately the C6-C7 level, and the projected image from the navigated wand was used to approximate the screw trajectory. If the patient's anatomy and body habitus allowed for C1-C2 transarticular screw fixation, a navigated pilot hole was made just above the $\mathrm{C} 2-\mathrm{C} 3$ lateral mass in the midline of the lateral mass. Pilot hole placement was followed by navigated drilling, tapping, and screw placement through the $\mathrm{C} 2$ pars, across the $\mathrm{C} 1-2$ joint, and into the $\mathrm{C} 1$ lateral mass. The $\mathrm{C} 1-\mathrm{C} 2$ transarticular did not require a tulip. If a $\mathrm{C} 1-\mathrm{C} 2$ transarticular screw trajectory was not feasible or desirable, C1 lateral mass and $\mathrm{C} 2$ pars screw/rod fixation was accomplished by modifying this technique. Shorter C2 pars screws with tulips were placed in the fashion described above. Separate bilateral stab incisions were made directly above $\mathrm{C} 1$ lateral masses and pilot hole placement was followed by navigated drilling, tapping, and screw placement directly into the $\mathrm{C} 1$ lateral masses. Extended tab screws were not required. Rods were then placed directly into the C1-C2 screw tulips and locking caps are engaged.

\section{Technical Note}

The soft tissue and fascia surrounding the lateral masses can cause significant difficulty with cannulating the initial pilot hole. To prevent this from occurring, it is important to ensure there is an appropriate corridor through the lateral neck musculature and fascia. If there is any resistance encountered with cannulating the entry point or obtaining screw purchase, we recommend reorienting with the navigated wand and widening any muscle and fascia near the lateral mass entry point.

To reduce the catastrophic risk of bilateral vertebral artery injury, screws should be placed on 1 side at a time. If there is any concern for significant breach or inaccurate navigation, an intraoperative CT should be performed prior to proceeding with the contralateral side to confirm there has not been significant foraminal breach and potential vertebral artery compromise.

In cases of traumatic instability, especially with C1-C2 bony and ligamentous injury, special care must be taken to avoid exerting undue pressure onto the spine during the drilling and tapping portions of the procedure to avoid inaccurate screw placement. The C2 pars screws should be placed prior to $\mathrm{C} 1$ lateral mass screw placement. Exacting pilot hole placement and soft tissue release facilitates accurate screw placement.

Rods should not be passed until all screws have been placed. Due to the significant ligamentous laxity in the cervical spine and the small margin of error, placement of the rod can reduce the accuracy of the navigation on the contralateral side.

\section{RESULTS}

From January 2015 to May 2020, a total of 27 consecutive patients who underwent navigated MIS posterior cervical fixation at 2 separate sites were evaluated. Indication for operations included type II odontoid fracture, subaxial fracture dislocation and traumatic burst fracture, metastatic pathological burst fracture, and degenerative spondylosis with stenosis. There were 15 men and 12 women, with an average age 63.5 years. Follow-up ranged from 3 to 24 months (average $=16.7$ months). Eleven patients underwent anterior cervical surgery followed by staged posterior MIS fixation. The remaining 16 patients underwent posterior-only MIS fixation with or without decompression. On average, 4 levels were included in the posterior construct. One screw was revised intraoperatively. Two patients $(7.7 \%)$ required another operation, 1 patient required repositioning of a $\mathrm{C} 5$ pedicle screw, and 1 suffered a $\mathrm{C} 7$ body fracture. No nerve root injury, spinal cord injury, or vertebral artery injuries or surgical site infections occurred in this cohort.

Overall, there were 3 screws revised for malpositioning, 2 intraoperative and 1 postoperative. The 2 intraoperative repositionings occurred after confirmation intraoperative imaging showed suboptimal screw placement. Both screws were repositioned using navigation guidance without sequelae. There was concern over the C5 screw impinging on the foramen transversarium and potentially the vertebral artery. The screw was removed and replaced with a more medial trajectory; there was no vascular compromise evident. The $\mathrm{C} 2$ screw was noted to be 
placed cephalad in the $\mathrm{C} 2$ pars with poor screw purchase. It was repositioned more caudally and medially with good screw purchase; there was noted to be no neurologic or vascular compromise. The 1 postoperative screw repositioning occurred after the patient recovered from anesthesia and complained of shoulder pain. Postoperative CT imaging revealed a screw impinging on the C4-C5 foramen. The patient returned to the operating room, where the screw was removed and not replaced. The patient's pain resolved without neurologic deficit.

There was virtually no radiation exposure to the surgical team as they broke scrub and left the operating room suite during intraoperative $\mathrm{O}$-arm imaging. The patient was exposed to $1 \mathrm{O}$-arm spin for screw navigation. The initial several patients in each of the senior surgeons' experience were exposed to a second $\mathrm{O}$-arm spin for verification of instrumentation placement. The second verification O-arm spin was replaced by plain radiograph verification imaging later in the series.

\section{DISCUSSION}

Cervical pedicle screws have been shown to provide significant biomechanical advantage over the more commonly performed lateral mass fixation. ${ }^{3-5}$ The narrow pedicle size and concern for potential breach leading to injury to the spinal cord, nerve root, and vertebral artery have limited its use. Several studies have demonstrated that the actual rate of complication with traditional open cervical pedicle screw placement is very low. ${ }^{12,13}$

In the largest cervical pedicle screw study to date, Abumi et al ${ }^{13}$ described their complication rate with 180 patients undergoing open placement of 712 total cervical pedicle screws. Complications included 2 nerve root injuries by screw $(0.28 \%), 1$ nerve root injury secondary to foraminal stenosis $(0.14 \%)$, and 1 vertebral artery injury by screw $(0.14 \%)$, for a total neurovascular complication rate of $0.5 \%$. No spinal cord injuries were reported. This was shown to be similar in complication rate to the largest series of lateral mass fixations. ${ }^{14-18}$

Open posterior cervical stabilization with lateral mass fixation or traditional cervical pedicle screw technique requires significant disruption of the posterior musculoligamentous tension band. This contributes significantly to postoperative neck muscle dysfunction and atrophy with concomitant neck pain. ${ }^{6}$ The advantages of percutaneous MIS techniques for lumbar spine surgery with regard to muscle-sparing approaches are well documented. ${ }^{7,19}$ To achieve similar results in the posterior spine, a true percutaneous technique is required.

Current literature of percutaneous cervical pedicle screws is limited to mostly $\mathrm{C} 2$ and $\mathrm{C} 7$ pedicle screws, which are traditionally much larger in diameter and present a reduced risk of vertebral artery injury. ${ }^{20}$ There are small series of reported percutaneous assisted cervical pedicle screws with traditional open exposure. The largest true percutaneous cervical series was performed fluoroscopically in 15 patients. $^{21}$ Screw placement using fluoroscopy typically requires the use of K-wires and/or tubular or cylindrical retractors, which can limit screw trajectory and potentially complicate the procedure. $^{22-24}$ The adoption of advanced intraoperative $\mathrm{CT}$ and navigation techniques simplifies the percutaneous pedicle screw placement, making it more accurate and reproducible without the need for specialized retractors or intraoperative fluoroscopy.

\section{Potential Indications}

The MIS, navigated percutaneous cervical technique described in the present article can be used for stand-alone posterior cervical fixation or in conjunction with MIS or open decompression. It can be used in conjunction with anterior cervical decompression as part of a single surgery or a staged anterior-posterior procedure. It can also be adapted for use with intraoperative robot-assisted navigation. From our early experience, we have identified patients who would potentially benefit most from implementation of this posterior MIS technique. The use of percutaneous cervical pedicle screws from a supplemental perspective following anterior surgery helps reduce the approach-related morbidity of a combined anterior-posterior operation. Patients with a high risk of wound healing issues, such as multi-trauma patients, may benefit substantially from this technique. Avoiding a large midline incision, with significant potential for the dead space of traditional approaches, is especially advantageous in patient populations with spinal cord injury, osteomyelitis, metastatic tumor, or end-stage renal disease. . $^{7,25,26}$

Patients undergoing anterior cervical corpectomy with significant risk of construct failure may benefit from this posterior MIS technique, especially patients with osteoporosis, metastatic disease, infection, or end-stage renal disease who 
otherwise may not receive posterior stabilization due to approach-related morbidity. Patients who have undergone multi-level corpectomy are also potential candidates for percutaneous stabilization.

Cervical fracture dislocation is another indication for this technique. After reduction of the fracture with traction with or without addition of anterior decompression and fusion, percutaneous pedicle screw fixation can be used as a primary or secondary means of fixation. In addition, unstable cervical spine fractures with preserved alignment and no spinal cord compression may also benefit from this technique. We have found this technique particularly useful in the subset of patients with cervical spine diffuse idiopathic skeletal hyperostosis fractures. Two patients with diffuse idiopathic skeletal hyperostosis with unstable cervical fractures in our series were able to be reduced and decompressed anteriorly with a 1-level anterior cervical discectomy and fusion and then received long-segment fixation posteriorly via this technique. Acute or chronic type II odontoid fractures, especially in the elderly, represent another patient population amenable to this MIS approach.

This study reports the initial experience with this technique for all primary surgeons and therefore reflects the expected learning curve as it relates to the radiographic and clinical outcomes. In addition, this technique should be reserved for spinal surgeons who have significant open and MIS navigation experience in the thoracic and lumbar spine. Intraoperative navigation alone requires a separate learning curve, and it is not recommended that comfort with it be built with this technique. The primary surgeons in this study each had at least 5 years of experience with intraoperative CTguided navigation prior to implementing this technique.

The limitations of this report on our initial experience with a novel MIS percutaneous technique include retrospective nature of the study as well as the relatively small number of patients and the lack of clinical correlation with validated patient-reported outcome measures. This report is intended to be a feasibility and safety analysis with radiographic outcome. Future, prospective study should focus on a comparative analysis to open posterior cervical procedures including clinical outcomes and economic measures.

\section{CONCLUSION}

Percutaneous posterior cervical pedicle screw fixation is a feasible and safe technique when performed with advanced intraoperative navigation. This technique should be reserved for spine surgeons with significant navigation experience, given the potential complications of pedicle screw breach in the cervical spine. Cervical pedicle screw fixation provides a biomechanically superior construct in comparison with lateral mass technique. In addition, the lack of paraspinal muscle disruption associated with the muscle-sparing approach preserves important stabilizers of the posterior ligamentous complex and may reduce wound-healing issues in high-risk patients. Furthermore, the robust biomechanical fixation coupled with the musclesplitting approach allows for earlier mobilization without the need for external bracing. Whereas the current role for percutaneous instrumentation is relatively narrow, the advancement of minimally invasive cervical decompressive techniques ${ }^{8,9,27}$ and intraoperative navigation technologies, including robotics, may provide expanded opportunities in the future.

\section{REFERENCES}

1. Wang MY, Levi ADO. Minimally invasive lateral mass screw fixation in the cervical spine: initial clinical experience with long-term follow-up. Neurosurgery. 2006;58(5):907-912, discussion 907-912. doi:10.1227/01.NEU.0000209929.38213.72

2. Sharan A, Hardenbrook M, Ratliff J, Harrop J. MIS Posterior cervical spine surgery: five-level fusion through a novel cervical tube. JHN Journal. 2011;6(2). doi:10.29046/ JHNJ.006.2.004

3. Schmidt R, Wilke H-J, Claes L, Puhl W, Richter M. Pedicle screws enhance primary stability in multilevel cervical corpectomies: biomechanical in vitro comparison of different implants including constrained and nonconstrained posterior instrumentations. Spine. 2003;28(16):1821-1828. doi:10.1097/ 01.BRS.0000083287.23521.48

4. Kowalski JM, Ludwig SC, Hutton WC, Heller JG. Cervical spine pedicle screws: a biomechanical comparison of two insertion techniques. Spine. 2000;25(22):2865-2867. doi:10. 1097/00007632-200011150-00005

5. Richter M, Wilke HJ, Kluger P, Neller S, Claes L, Puhl W. Biomechanical evaluation of a new modular rod-screw implant system for posterior instrumentation of the occipitocervical spine: in-vitro comparison with two established implant systems. Eur Spine J. 2000;9(5):417-425. doi:10.1007/ s005860000173

6. Bodon G, Patonay L, Baksa G, Olerud C. Applied anatomy of a minimally invasive muscle-splitting approach to posterior C1-C2 fusion: an anatomical feasibility study 2014. doi:10.1007/s00276-014-1274-x

7. Sugimoto Y, Hayashi T, Tokioka T. Minimally invasive 
cervical pedicle screw fixation via the posterolateral approach for metastatic cervical spinal tumors. Spine Surg Relat Res. 2017;1(4):218-221. doi:10.22603/ssrr.1.2016-0025

8. Adamson TE. Microendoscopic posterior cervical laminoforaminotomy for unilateral radiculopathy: results of a new technique in 100 cases. J Neurosurg. 2001;95(suppl 1):51-57. doi:10.3171/spi.2001.95.1.0051

9. Coric D, Adamson T. Minimally invasive cervical microendoscopic laminoforaminotomy. Neurosurg Focus. 2008;25(2):E2. doi:10.3171/FOC/2008/25/8/E2

10. Helm PA, Teichman R, Hartmann SL, Simon D. Spinal navigation and imaging: history, trends, and future. IEEE Trans Med Imaging. 2015;34(8):1738-1746. doi:10.1109/TMI. 2015.2391200

11. Chachan S, Bin Abd Razak HR, Loo WL, Allen JC, Shree Kumar D. Cervical pedicle screw instrumentation is more reliable with $\mathrm{O}$-arm-based $3 \mathrm{D}$ navigation: analysis of cervical pedicle screw placement accuracy with O-arm-based 3D navigation. Eur Spine J. 2018;27(11):2729-2736. doi:10.1007/ s00586-018-5585-1

12. Ito Y, Sugimoto Y, Tomioka M, Hasegawa Y, Nakago K, Yagata Y. Clinical accuracy of 3D fluoroscopy-assisted cervical pedicle screw insertion. J Neurosurg Spine. 2008;9(5):450-453. doi:10.3171/SPI.2008.9.11.450

13. Abumi K, Shono Y, Ito M, Taneichi H, Kotani Y, Kaneda K. Complications of pedicle screw fixation in reconstructive surgery of the cervical spine. Spine. 2000;25(8):962-969. doi:10.1097/00007632-200004150-00011

14. Fehlings MG, Cooper PR, Errico TJ. Posterior plates in the management of cervical instability: long-term results in 44 patients. J Neurosurg. 1994;81(3):341-349. doi:10.3171/jns. 1994.81.3.0341

15. Graham AW, Swank ML, Kinard RE, Lowery GL, Dials BE. Posterior cervical arthrodesis and stabilization with a lateral mass plate: clinical and computed tomographic evaluation of lateral mass screw placement and associated complications. Spine. 1996;21(3):323-328.

16. Heller JG, Silcox DHI, Sutterlin CEI. Complications of posterior cervical plating. Spine. 1995;20(22):2442-2448.

17. Levine AM, Mazel C, Roy-Camille R. Management of fracture separations of the articular mass using posterior cervical plating. Spine. 1992;17(10S):S447. doi:10.1097/ 00007632-199210001-00018

18. Wellman BJ, Follett KA, Traynelis VC. Complications of posterior articular mass plate fixation of the subaxial cervical spine in 43 consecutive patients. Spine. 1998;23(2):193-200.

19. Kim D-Y, Lee S-H, Chung SK, Lee H-Y. Comparison of multifidus muscle atrophy and trunk extension muscle strength: percutaneous versus open pedicle screw fixation. Spine. 2005;30(1):123-129.

20. Karaikovic EE, Daubs MD, Madsen RW, Gaines RW. Morphologic characteristics of human cervical pedicles. Spine. 1997;22(5):493-500. doi:10.1097/00007632-199703010-00005

21. Schaefer C, Begemann P, Fuhrhop I, et al. Percutaneous instrumentation of the cervical and cervico-thoracic spine using pedicle screws: preliminary clinical results and analysis of accuracy. Eur Spine J. 2011;20(6):977-985. doi:10.1007/s00586011-1775-9

22. Holly LT, Foley KT. Percutaneous placement of posterior cervical screws using three-dimensional fluoroscopy. Spine. 2006;31(5):536-540, discussion 541. doi:10.1097/01.brs. 0000201297.83920.a1

23. Holly LT, Isaacs RE, Frempong-Boadu AK. Minimally invasive atlantoaxial fusion. 2010. doi:10.1227/01.NEU. 0000366107.69895 .74

24. Joseffer SS, Post N, Cooper PR, Frempong-Boadu AK. Minimally invasive atlantoaxial fixation with a polyaxial screwrod construct: technical case report. Neurosurgery. 2006;58(4 suppl 2):ONS-E375, discussion ONS-E375. doi:10.1227/01. NEU.0000208955.12461.C7

25. Oda I, Abumi $\mathrm{K}$, Ito $\mathrm{M}$, et al. Palliative spinal reconstruction using cervical pedicle screws for metastatic lesions of the spine: a retrospective analysis of 32 cases. Spine. 2006;31(13):1439-1444. doi:10.1097/01.brs.0000219952.40906. $1 \mathrm{f}$

26. Neo M, Sakamoto T, Fujibayashi S, Nakamura T. The clinical risk of vertebral artery injury from cervical pedicle screws inserted in degenerative vertebrae. Spine. 2005;30(24):2800-2805. doi:10.1097/01.brs.0000192297.07709. $5 \mathrm{~d}$

27. Hussain I, Schmidt FA, Kirnaz S, Wipplinger C, Schwartz TH, Härtl R. MIS approaches in the cervical spine. J Spine Surg. 2019;5(suppl 1):S74-S83. doi:10.21037/jss.2019. 04.21

Disclosures and COI: The authors have no personal or institutional financial interest in the drugs, materials, or devices described in this article, except as follows: Domagoj Coric, MD: Spine Wave - consultant, royalties, direct stock ownership; and Medtronic - consultant, royalties; Timothy Adamson, MD: Spine Wave-consultant, royalties; John Peloza, MD: Spine Wave - consultant, royalties. The other authors report no conflicts of interest concerning the materials or methods used in this study or the findings specified in this article.

Corresponding Author: Vincent Rossi, MD, Atrium Health Musculoskeletal Institute, Carolina Neurosurgery and Spine Associates, 225 Baldwin Ave, Charlotte, NC 28204. Phone: (309) 339-0816; Email: Vrossi89@gmail.com.

Published 9 December 2020

This manuscript is generously published free of charge by ISASS, the International Society for the Advancement of Spine Surgery. Copyright (c) 2020 ISASS. To see more or order reprints or permissions, see http://ijssurgery.com. 